\title{
EVALUATING DIFFERENT WATER-LAND-BOUNDARY APPROXIMATIONS TO IMPROVE SAR-DERIVED DIGITAL ELEVATION MODELS
}

\author{
B. Schneider ${ }^{1}$, E. Wortmeyer ${ }^{2}$, S. Schmitz ${ }^{3,4}$, A. Thiele ${ }^{3,4}$, P. Havel $^{1}$, H. Dirks ${ }^{2}$ \\ ${ }^{1}$ German Federal Institute of Hydrology, Koblenz, Germany - Bastian.Schneider@bafg.de \\ ${ }^{2}$ Lower Saxony Water Management, Coastal Defence and Nature Conservation Agency, \\ Coastal Research Station, Norden, Germany \\ ${ }^{3}$ Institute of Photogrammetry and Remote Sensing, Karlsruhe Institute of Technology, Karlsruhe, Germany \\ ${ }^{4}$ Fraunhofer IOSB, Ettlingen, Institute of Optronics, System Technologies and Image Exploitation, Germany
}

Commission I, WG I/3

KEY WORDS: SAR, RTK, GNSS, ALS, WATER-LAND-BOUNDARY, SEA-LEVEL, GERMAN WADDEN SEA, TIDAL FLATS.

\begin{abstract}
:
The coastline of the German Wadden Sea is constantly subjected to the tides and the tidal-induced environmental changes like erosion and accumulation of sediments need to be monitored constantly. This task requires digital elevation models (DEMs), which are derived from remote sensing data. To model those DEMs, a separation of data collected over landmasses and water bodies is required. In the GeoWAM project the potential of airborne SAR-data (F-SAR) is investigated for monitoring purposes in the Wadden Sea. As part of the project, this paper focuses on the suitability of F-SAR data regarding the derivation of water-landboundaries (WLBs). Therefore, water-land-boundaries based on independent data sets are compared and evaluated. Analyzed data sets include data collected via F-SAR, airborne laserscanning (ALS), on site GNSS measured WLB points and sea-level data from two acoustic gauges. The algorithms were tested on a study site on Spiekeroog island. Our results show, that the accuracies of the derived WLBs mostly depend on the on-site topography and sediments. The spatial deviation between the reference data and the approximated WLBs is mostly less than $2 \mathrm{~m}$ horizontally and $0.15 \mathrm{~m}$ vertically. Identified challenges to overcome are mostly related to processing of F-SAR data in areas with highly water saturated sediments. Our results suggest, that F-SAR data in tidal flats is not necessarily dependent on further supplementing surveys, as one of the main advantages of the F-SAR data is the potential to derive DEMs and WLBs from the same data set.
\end{abstract}

\section{INTRODUCTION}

The German Wadden Sea is a highly dynamic environment, which is constantly subjected to the tides. The necessity to monitor tidal-induced changes like erosion and accumulation of sediments and the associated changes of topography and coastlines rely on frequently updated high-resolution digital elevation models (DEMs). Important applications depending on those DEMs are e.g. marine navigation, environmental monitoring or coastal protection. Currently, airborne laserscanning (ALS) is widely used for coastal DEM generation (Dorninger, 2011), but limitations of ALS (weather dependency, limited swath, flightline fusion) hamper further development in tidal areas (Bolz et al., 2020). Airborne SAR-data (F-SAR) is expected to overcome typical problems of ALS in tidal areas and aims to complement ALS data in the future (Horn et al., 2017). Improvements are expected due to a much higher swath and footprint at a comparable spatial resolution, complemented by high resolution multiband data sets (Pinheiro et al., 2020b).

The GeoWAM project is developing and testing F-SAR systems to create high-resolution SAR-DEMs (Pinheiro et al., 2020a) and a wide range of other environmental applications (Schmitz et al., 2021). In order to model fused SAR-DEMs of tidal areas (DEM-W), land (F-SAR) and water (sonar) data need to be integrated, and to do so, accurate water-land-boundaries (WLBs) are essential (Sellerhoff, 2011).

\footnotetext{
* Corresponding author
}

Since mudflats are only exposed around low tide, available time windows for data acquisition are very short and limited. Therefore, especially airborne SAR systems that are capable of taking high-resolution images regardless of cloud cover and weather conditions represent an optimal tool for surveying. However, even at low tide mudflats are interfused by a network of waterfilled tidal creeks.

Although SAR systems have a wider swath than ALS systems and therefore an advantage in terms of acquisition time, the data acquisition is carried out in multiple flight stripes. Between adjacent stripes the water level during acquisition can vary significantly, which leads to complex land-water boundaries and the question, how this border can be described reasonably (Sellerhoff, 2011). Especially in the transition zone between two flight stripes, the separation between points associated with land and water is difficult. If the same amount of time is considered, the variation in water level will be less in areas with steep shores (sheet pile) than in areas with shallow banks (tidal flat, beaches). As described by (Sellerhoff, 2011), in some cases a continuous land-water boundary and in others a disjoint amount of individual polygons, which include jumps, will be the best choice to describe the dynamic land water surface relation. The derived boundary is considered to be a $2 \mathrm{D}$ separator, but data points collected over water bodies correspond to the water surface. Taking advantage of this, the height of the landwater boundary can be determined, which yields a 3D interpolation line that can be used during the digital elevation modeling (Dorninger, 2011). 
This study analyzes the currently achievable deviations and accuracies of different WLB data sets in respect to the reference RTK-GNSS survey points. Compared to reference data we aim at a maximum spatial deviation of $\leq 2 \mathrm{~m}$ horizontally and $\leq 0.15$ m vertically. Knowledge on the accuracy and suitability of F-SAR-dependent and independent sea-level reference data will contribute significantly to establish improved algorithms to generate WLBs from F-SAR-data and improve the overall DEM-generation in tidal areas.

\section{STUDY AREA}

\subsection{The Wadden Sea}

The Wadden Sea is a large intertidal zone in the south-eastern part of the North Sea in the German Bight (see Figure 1). Its coastline stretches over $500 \mathrm{~km}$ from the Netherlands in the west, along the German northwestern coastline, up to southwestern Denmark and is interrupted by a number of river estuaries, most importantly the Elbe river estuary. Parallel to the coastline, the chain of the Frisian Islands forms a natural protecting barrier for dry falling mud flats between the islands and the coastline of the mainland. The total area encompasses over $10.000 \mathrm{~km}^{2}$, making the Wadden Sea the largest tidal sea worldwide.

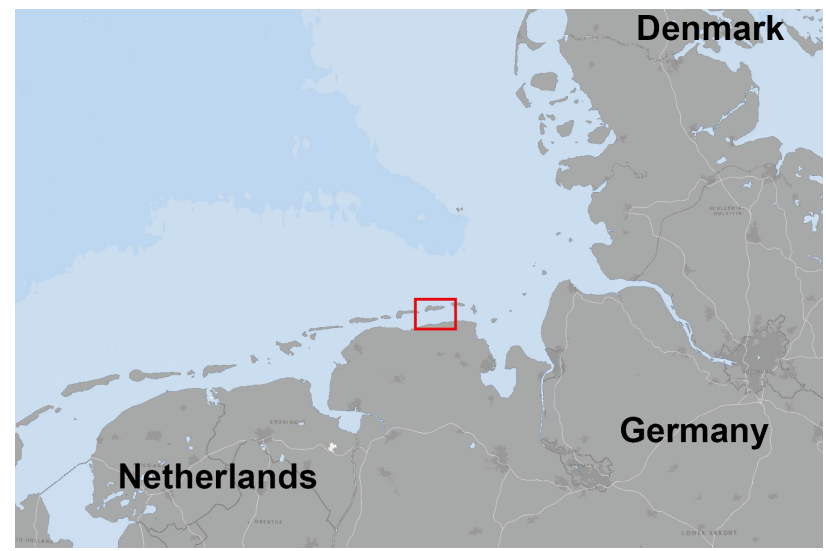

Figure 1. Location of the Wadden Sea in the German Bight. The chain of the Frisian Island can be traced off-shore the mainland with the island of Spiekeroog in a red box.

The Wadden Sea is characterized by a shallow water body, lowlying tidal flats and wetlands, interrupted by a dense dendritic network of tidal creeks. Wind and wave impact, as well as tidal currents constantly shift the predominately silty to sandy sediments of the Wadden Sea, ever-changing the course of the coastline as well as moving the Frisian Islands eastwards. Nowadays, the exposed, western ends of the islands are fixated. The Wadden Sea is characterized by significant tidal activities. The tidal regime is semi-diurnal (two high tides, two low tides per day), while the tidal range rises from west to east. The island of Spiekeroog has a tidal range of about $2 \mathrm{~m}$, indicating a meso-tidal system.

The high biodiversity of the Wadden Sea and its unique flora and fauna led to the establishment of three national parks in Germany, which were added to the World heritage List of UNESCO in 2009. Beyond that, the area is characterized by a long history of human intervention and the everlasting struggle of land reclamation, prevention of erosion and economic pressures on the environment, resulting from activities such as fishing, marine traffic and tourism.

\subsection{The island of Spiekeroog}

The site which we used to evaluate the different WLBs is located on the island of Spiekeroog. Spiekeroog is part of the Frisian Island chain and located in the center of the Wadden Sea coastline. The island itself is an erosional remain of sand dunes and sandbars. Due to tides and the predominately westerly winds, the island itself is constantly shifting its form. Spiekeroog is about $10 \mathrm{~km}$ long, up to $2.5 \mathrm{~km}$ wide and located about $6 \mathrm{~km}$ offshore the mainland in a northern direction. In between Spiekeroog, the mainland and the neighboring islands, an extensive network of tidal creeks of various size channel the tidal water flow. Smaller tidal creeks usually fall dry during low tide, while the bigger tidal creeks typically remain flooded.

A system of man-made coastal management structures, including levees, sea walls or groynes, but also natural barriers such as stabilized dune ridges protect the populated parts of the island from flooding. These structures are especially important in the western part of the island, as coastal erosion is most intense here due to the exposition to westerly winds and currents.

The study site, where we evaluate the different WLBs is located on a beach at the westernmost tip of Spiekeroog island (see Figure 2). The beach is confined by two large, rocky groynes, about $170 \mathrm{~m}$ wide and gently dips seawards with a very shallow angle. The sediments on the study site are dominantly sandy, with patches of finer grained silty to clayey material on the more shielded parts of the beach next to the groynes.

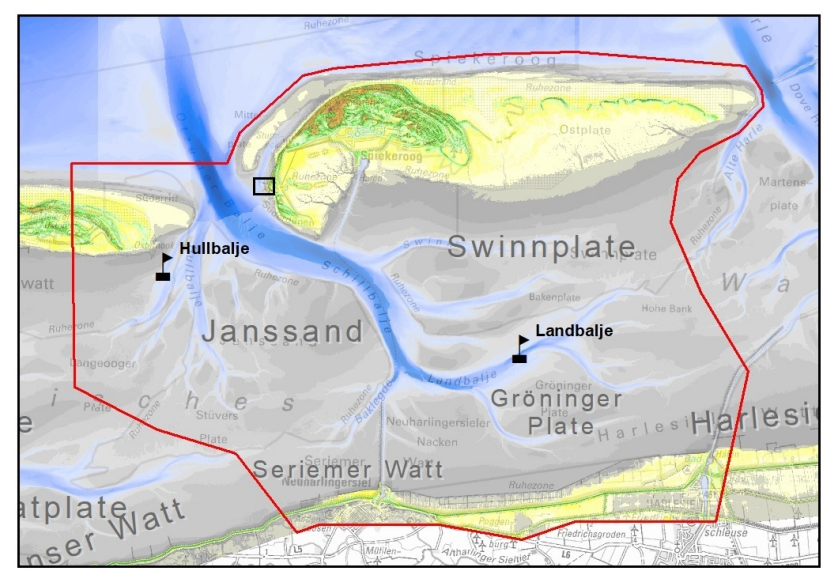

Figure 2. F-SAR surveyed area (red frame) in the German Wadden Sea. The flags indicate the positions of the sea-level gauges. Location of the relevant study site on the western tip of Spiekeroog island (Figure 6) is indicated by a black outline.

\section{METHODS}

Acquisition of the various data sets compared in this study including F-SAR, ALS, GNSS measurements of the WLB and recordings of two adjacent sea-level gauges - were collected in August 2020 on the island of Spiekeroog in the German Wadden Sea (see Figure 2). The F-SAR-data acquisition of the study site was carried out on the 23rd of August 2020 during low-tide between 06:45 and 06:57 am UTC, while GNSS WLB data was 
recorded between 05:57 am UTC, and 07:26 am UTC simultaneously on the same day.

The ALS survey of the study area was commissioned a week prior to the F-SAR-survey while the two sea-level gauges are recording data continuously throughout the entire period.

\subsection{GNSS data acquisition}

In this study, our GNSS-surveyed WLB points are considered as reference data points, hence we compare the other data sets in respective to GNSS data. GNSS data sets were collected on site with a focus on pinpointing the WLB as precisely as possible. The GNSS-WLB points were collected using a Trimble R10 GNSS receiver mounted on a monopod and a Trimble TSC3 GNSS controller in the "topography point" mode resulting in a sub-centimeter accuracy of the reference points. Points were continuously recorded on the $170 \mathrm{~m}$ wide beach between the two groynes during the entire F-SAR flight with two devices, keeping a distance of about $20 \mathrm{~m}$ between each recorded data point. Each WLB data point was recorded at the highest point of the onshore swash of a wave, typically indicated by a thin line of sea foam as shown in Figure 3.

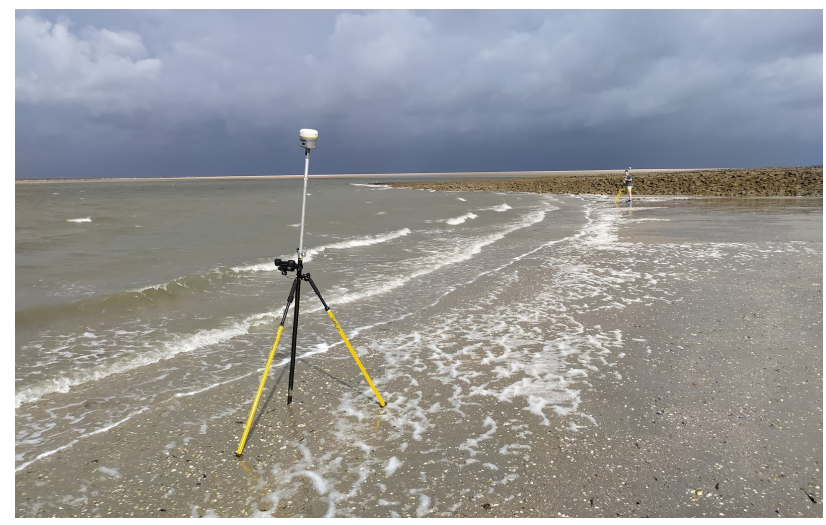

Figure 3. GNSS survey during the F-SAR flight mission.

Direction of view towards north. Note the (in this case already scattered) sea foam as indicator for the WLB.

Typical on-site challenges while recording the WLB are due to the changing wind and wave parameters during the survey campaign. At the time of the data acquisition, winds with a maximum wind speed of $25 \mathrm{~km} / \mathrm{h}$ and gusts from a southwestern - and therefore landwards - direction dominated. The wind and gusts resulted in slightly varying wave heights and therefore swash uprush onshore over the time of the survey. Additionally, bow waves and surges from passing vessels have the potential to superimpose the location of the WLB by a couple of meters. However, on this study site no large vessels passed by during the campaign. In other areas, such as the neighboring Elbe estuary with a high number of large container vessels, bow wave induced WLB shifting needs to be accounted. Additionally, errors in the vertical location of the surveyed WLB points can be caused by the sinking of the monopod in areas with highly liquefied and soft sediments.

In total, we recorded 283 WLB points. For the calculation of the reference WLB, we only used points from 10 minutes before the F-SAR overpass until 10 minutes after the overpass of the F-SAR.

\subsection{SAR data acquisition and modeling}

SAR data were acquired using the airborne F-SAR system, mounted on a Dornier Do228 aircraft and flying at an average altitude of $2440 \mathrm{~m}$. The F-SAR system is capable of acquiring fully polarimetric data in multiple frequency bands (X-, C-, S-, L- and P-bands) simultaneously (Horn et al., 2009). Additionally, X- and S-band antennas are able to perform single-pass polarimetric interferometry measurements. Repeat-pass PolInSAR measurement additionally serves as the standard measurement mode to ensure baseline flexibility. With a resolution of up to $25 \mathrm{~cm}$ in X-band, the F-SAR system provides high spatial resolution data. In the conducted measurement campaign, fully polarimetric data were acquired in the S- and L-band and VV-polarized data were acquired in the X-band. Further acquisition parameters are given in (Pinheiro et al., 2020b). The polarimetric image of the study area is depicted in Figure 4. Interferometric measurements are conducted in repeat-pass configuration and additionally with the single-pass S- and X-band interferometers. The interferometric SAR data sets are used to generate a DEM (1 m raster) (Pinheiro et al., 2020b).

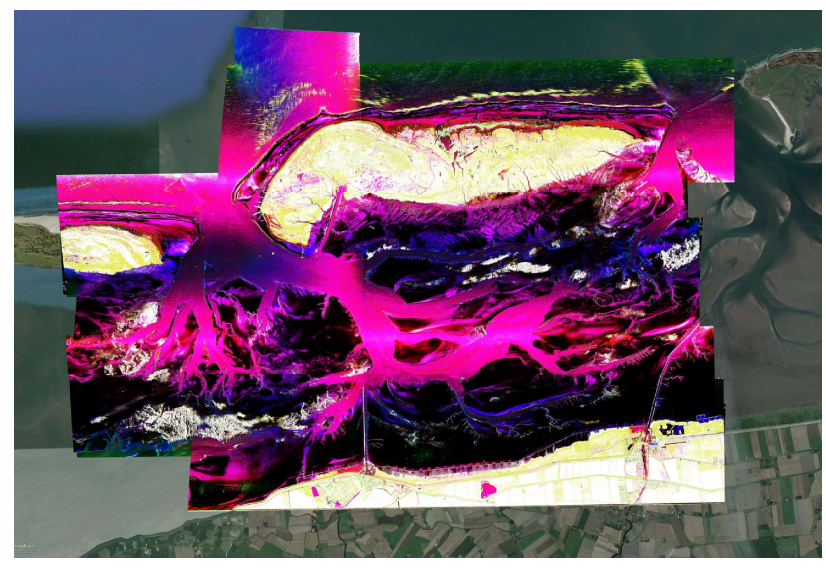

Figure 4. Polarimetric composite based on S-band data of the study site.

Furthermore, a mask was generated that separates water and land areas, based on the S-band data. The distinguishing features are VV polarized amplitudes from the master and slave images of the repeat-pass measurement in the S-band and the interferometric coherence between these two co-registered images. Figure 5 shows the amplitude and the coherence image for the selected study site. Water surfaces are characterized by low amplitudes as well as low coherence values. The low amplitude is due to the fact that water reflects the incident radar pulse away from the sensor. Moreover, the reflective property of the water surfaces leads to the complete decorrelation in repeat-pass interferograms, therefore the interferometric coherence is suitable as a distinguishing feature. Using appropriate thresholds, the determination of which is described in (Pinheiro et al., 2020b), each pixel is assigned to the class water or land, resulting in a 2D WLB, denoted as IMASKE.

\subsection{ALS data acquisition}

ALS data acquisition was performed on 16th of August 2020, using a Cessna C208 platform and a Riegl VQ 780i laserscanner at an average flight altitude of $1220 \mathrm{~m}$ above ground. The flight mission was carried out during low tide, between 11:45 and 15:50 UTC. The resulting ALS data set is characterized by 


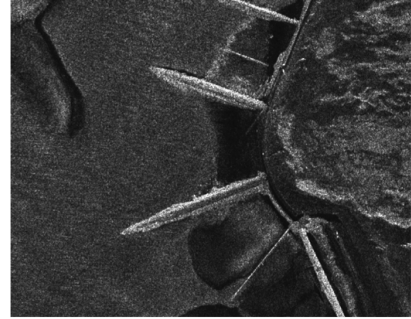

(a)

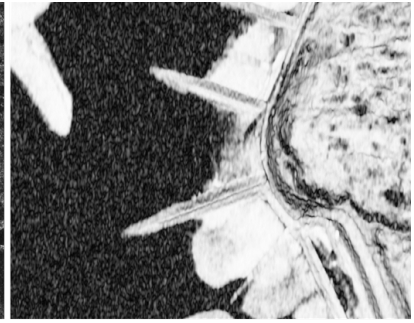

(b)
Figure 5. Distinguishing features for the generation of WLB: In (a) S-band VV amplitude (Master) and in (c) interferometric coherence (S-band, VV) is illustrated.

a point density of $\geq 3$ points $/ \mathrm{m}^{2}$ with a maximum point-topoint distance of $0.75 \mathrm{~m}$. Based on the ALS point cloud input, a rasterized DEM with a spatial resolution of $1 \mathrm{~m} \times 1 \mathrm{~m}$ was produced. As the ALS mission was commissioned seven days prior to the F-SAR mission, minor changes in the topography of the tidal flats and beaches due to tides, wave and weather action are to be expected. However, the weather on Spiekeroog was stable in the week between ALS and F-SAR acquisition, without significant winds, storms, tides or rain events, therefore no unusual and rapid changes in topography are assumed for that period.

\subsection{Gauge data acquisition}

Two sea-level gauges, both located on larger, permanently flooded tidal creeks between the island and the mainland (see Figure 2), were used to collect data on tidal sea-level variations in the study area. The sea-level gauge "Hull" is located about $3 \mathrm{~km}$ southwest of the study site, while the gauge "Land" is located further away, about $6.5 \mathrm{~km}$ southeast of the study site. The acoustic gauges collect data every $200 \mathrm{~ms}$ and bundle them into blocks of 100 measurements. If less than $75 \%$ of these measurements are accepted, the whole block is neglected. To identify invalid measuring points a filter is applied, which looks for too large discrepancies regarding the derivation. Measurements are categorized as outliers if the derivation between neighboring points exceeds $3 \mathrm{~m} / \mathrm{s}$. This way, events like spray or heavy rain can be excluded from the data set. To avoid outliers further a weighted mean minute value is calculated.

\subsection{WLB modeling}

To model WLBs as 3D-polylines, we rely on high resolution DEMs to plot the measured sea-level data on the local study site topography. Normally, we would utilize a F-SAR derived DEM, produced from the SAR data of the flight mission. However, the present F-SAR algorithms seemed to have problems with highly water saturated sediments in some areas, which we realized after analyzing our F-SAR DEM data sets. This resulted in a partly incomplete F-SAR DEM in the areas we intended to evaluate our WLB data sets. Due to this issue, we used the ALS-derived DEM with a spatial resolution of $1 \mathrm{~m} \times 1 \mathrm{~m}$ to create and evaluate the various $3 \mathrm{D}$ WLB data sets.

Apart from the ALS DEM, point data sets from GNSS, F-SAR derived 2D WLBs, sea-level data from the two gauges, and a WLB from ALS data were modeled. We used ESRIs ArcMap 10.5.1 to model the subsequent WLBs and calculate the spatial deviations between reference data and evaluated WLB data sets.
For details on input data acquisition, please refer to chapter 3.1 to chapter 3.4.

We used the GNSS measured WLB points 10 minutes before and 10 minutes after the overpass of the F-SAR to calculate an average GNSS sea-level at the time of the SAR data acquisition, resulting in an average water-level of $1.07 \mathrm{~m}$ below mean sealevel. An isoline at $1.07 \mathrm{~m}$ below mean sea-level was created, based on the ALS DEM input model, subsequently acting as the reference WLB together with the GNSS reference data. Other GNSS points were not considered in this study.

The ALS DEM was then used to allocate elevation data to the 2D IMASKE and create a 3D IMASKE. This was done by creating a dense string of points along the polyline and assigning them the respective elevation values of the ALS DEM. That is why, in contrast to the other WLBs, the IMASKE WLB has no constant elevation, but takes parameters as local wave shielding, onshore swash and effects of local topography into account.

As the ALS survey was a week prior to the other surveys, we had no immediate information on sea-level during the time of the ALS data acquisition. A pure ALS point cloud based water-land separation using information as return signal intensity and semi-supervised point cloud classification did not provide WLBs with a sufficient data quality. We therefore analyzed the 3D ALS point cloud of a cross-section of the study site with respect to changes in slope to pinpoint the location and elevation of the WLB at the time of the ALS survey. We assumed a flat surface for the water body and an onset of a gentle inclination at the WLB, where the beach topography begins to rise. By analyzing the changes in slope, we could pinpoint the elevation of the ALS WLB to $1.25 \mathrm{~m}$ below mean sea-level.

Consistent with the calculation of the GNSS WLB, we used the sea-level gauge data from 10 minutes prior to 10 minutes after the F-SAR overpass to calculate an average sea-level for each of the two gauges. The resulting average sea level for the gauge "Hull" was $0.96 \mathrm{~m}$ below mean sea-level and $1.01 \mathrm{~m}$ below mean sea-level for the gauge "Land". For both gauges, an isoline with the respective elevation was plotted on the ALS DEM.

The modeled courses of the individual WLBs as well as the location of GNSS reference data on the study site are depicted in Figure 6 below. After modeling the WLBs, the minimal horizontal and vertical distances from the GNSS points to every other WLB polyline were calculated in ArcMap 10.5.1. The horizontal and vertical deviation between the WLBs and GNSS reference data points were then used to qualitatively evaluate the various WLB approximations and data sets.

\section{RESULTS AND DISCUSSIONS}

Looking at the results of the survey mission in August 2020 reveals that the currently achievable WLB approximations fulfill the intended deviations from reference data (maximum spatial deviation of $\leq 2 \mathrm{~m}$ horizontally and $\leq 0.15 \mathrm{~m}$ vertically) in some parts already, while we observe rather large deviations in both directions in other parts of the study site (see Figure 7 and Table 1). We observe, that some WLBs (sea-level gauges, IMASKE in some cases) perform very well, while others (ALS, IMASKE in some areas) do not meet our requirements for various reasons yet. As we aim at delivering data products, which can be solely created from F-SAR data sets without the need of 


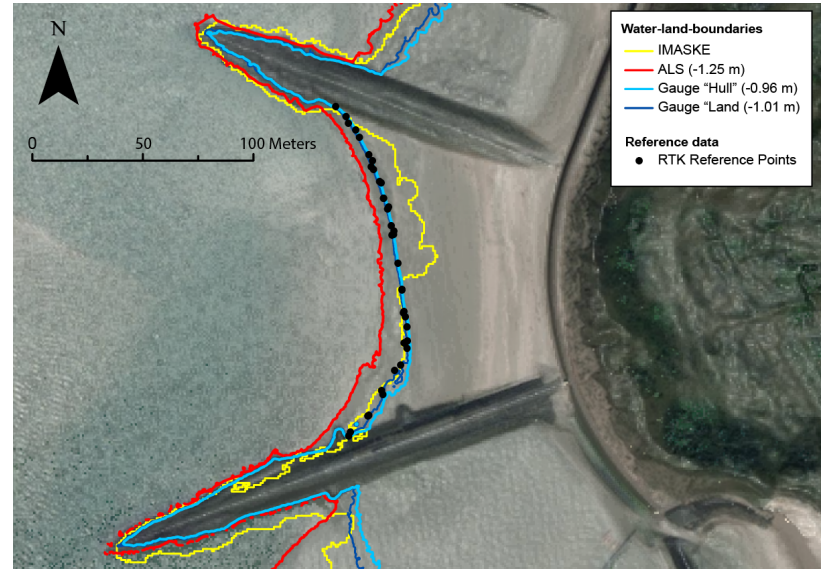

Figure 6. Study site with underlying ALS DEM, GNSS reference points and the evaluated WLB approximations. The location of the study site on Spiekeroog island is indicated in Figure 2.

other supplementary data sets from additional surveying methods, especially the WLB on base of the IMASKE is of importance here.

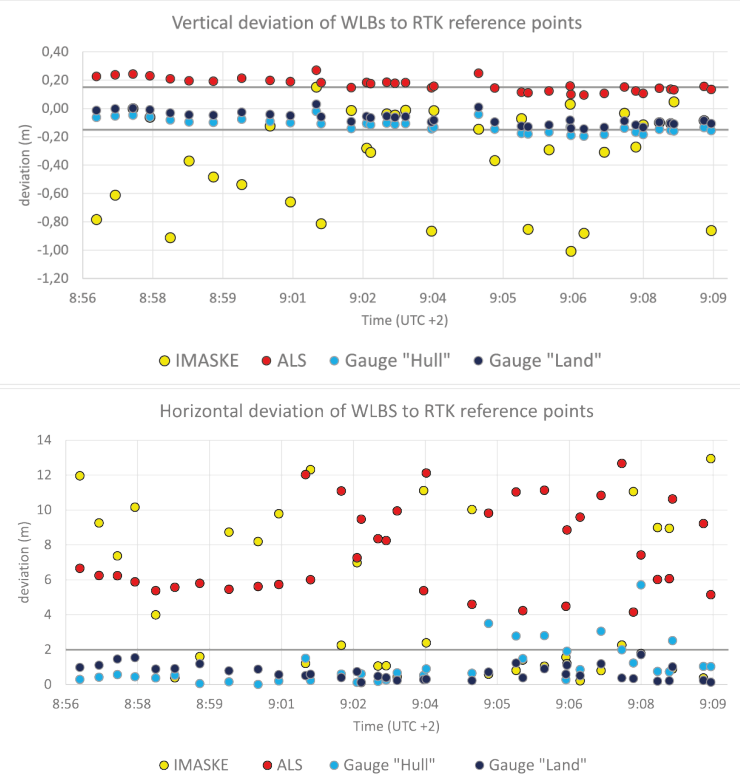

Figure 7. Vertical and horizontal deviations of WLBs to GNSS reference points. Tolerance lines are indicated.

The IMASKE predominantly delivers promising results (see Figure 7 and Table 1). While the deviations in the southern part of the study site are within or very close to the intended deviations, larger horizontal deviations exceeding $10 \mathrm{~m}$ in the northern part of the study area are evident (see Figure 6). As the deviation is limited to a small area and is not present in neighboring areas outside the study site, we can rule out significant problems in the IMASKE algorithm. We consider natural surface variations on the study site as the most likely reason for the onshore bulging of the WLB. Large areas with surficial water on the northern study site were documented at the end of the F-SAR survey (see Figure 8). We therefore assume that the surficial water and highly water saturated sediments in the northern parts of the study site currently exacerbate the clear delimitation of water and land areas using the IMASKE.

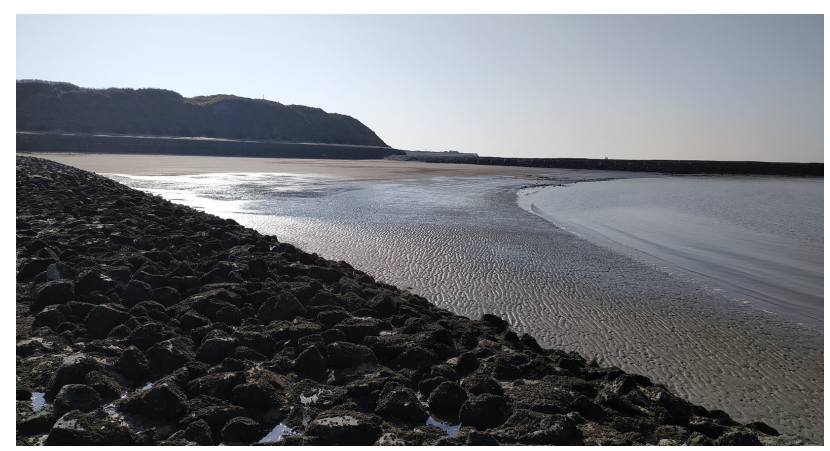

Figure 8. Photo of northern part of the GNSS survey site on Spiekeroog at the end of the F-SAR flight mission. Direction of view towards south. Note the surface water and water saturated patches in the left center.

The ALS data was recorded seven days prior to the F-SAR data and during a slightly lower water level in the tidal cycle. Therefore, the ALS WLB can not be directly transferred to the other data sets and has to be considered separately. Comparing the deviation of the ALS WLB with reference data and the other WLBs shows, that the vertical deviation in tidal flats is typically low $(\leq 0.3 \mathrm{~m})$ due to the very flat topography. Looking at horizontal ALS deviations clearly reveals - that in this case the ALS WLB shows large deviations from reference data of up to $13 \mathrm{~m}$ (see Figure 7). The impact of different water levels on deviations is furthermore amplified by the very low gradient of tidal flats and is therefore especially evident in the high horizontal deviations (see Table 1).

\begin{tabular}{lcccc} 
& IMASKE & ALS & Hull & Land \\
\hline \multicolumn{4}{c}{ Vertical deviation (in m) } \\
Average & $-0,33$ & 0,17 & $-0,12$ & $-0,07$ \\
Median & $-0,27$ & 0,16 & $-0,13$ & $-0,08$ \\
Standard deviation & 0,34 & 0,05 & 0,05 & 0,05 \\
Points within $<15 \mathrm{~cm}$ & 18 & 18 & 26 & 37 \\
$\%$ within $<15 \mathrm{~cm}$ & 48,6 & 48,6 & 70,3 & 100 \\
& \multicolumn{4}{c}{} \\
\hline \multicolumn{4}{c}{ Horizontal deviation (in m) } \\
Average & 4,73 & 7,68 & 1,10 & 0,68 \\
Median & 2,24 & 6,66 & 0,65 & 0,59 \\
Standard deviation & 4,42 & 2,53 & 1,19 & 0,42 \\
Points within $<2 \mathrm{~m}$ & 18 & 0 & 31 & 37 \\
$\%$ within $<2 \mathrm{~m}$ & 48,6 & 0 & 83,8 & 100 \\
\hline
\end{tabular}

Table 1. Vertical and horizontal deviation (in $\mathrm{m}$ ) of various WLBs in respect to the GNSS reference data

Looking at F-SAR-independent data, especially the tidal gauges deliver good results. As shown in Table 1, the recorded water levels of both gauges fit well with the GNSS reference data and mostly meet our requirements. Where available, local sea-level gauges seem to deliver high-quality data for WLB modeling in cases where no reference data is available and can help to evaluate the quality of SAR-derived WLBs. Notably, the WLB derived from the data of the more distant gauge "Land" fits slightly better than the WLB from the closer gauge "Hull". Gauge data is sensitive to local environmental conditions (e.g. tides, wind direction, surges), which emphasizes to consider gauge data with some caution and in context with environmental parameters at the time of data acquisition. 


\section{CONCLUSIONS}

By evaluating various methods to create reliable and accurate water-land boundaries, we demonstrated the potential of F-SAR to act as a new tool in topographical and environmental analysis in tidal areas. We tested F-SAR derived data against F-SAR independent data (ALS, sea-level gauges, GNSS) and demonstrated the currently possible accuracies of different WLBs. One of the main advantages and potentials of the F-SAR system is, that it allows deriving DEMs and WLBs from the same data set. This reduces or eliminates the need for supplementary methods such as local GNSS survey, sea-level gauges or ALS flights. Therefore, the F-SAR is especially suitable for remote study areas.

We identified remaining challenges to overcome in the future, which are mostly related to the processing of F-SAR data in areas with highly water saturated sediments. Firstly, the current F-SAR derived DEMs in those areas can be incomplete or patchy. This is why we used an ALS-derived DEM as an underlying elevation model in this study. Secondly, the high water saturation of the sediments currently hampers the derivation of highly accurate F-SAR-based WLBs. Refined algorithms for DEM and WLB generation from fully polarimetric F-SAR data are currently tested and implemented, promising improved results in the near future. Whether the addition of polarimetric and high level texture features within the classification process brings an improvement will be analyzed in the upcoming F-SAR missions. A first following survey was conducted in March 2021, where F-SAR and ALS flights were executed on the same day, reducing the time gap between data sets significantly and therefore creating much more comparable data sets and WLBs. Furthermore, an additional study site on the Elbe river estuary will allow us to apply, test and refine our algorithms on a much wider data basis and under different environmental conditions.

\section{ACKNOWLEDGEMENTS}

This study is part of the GeoWAM project, funded by the German Federal Ministry of Transport and Digital Infrastructure within the framework of the Modernity Fund ("mFUND").

\section{REFERENCES}

Bolz, T., Schmitz, S., Thiele, A., Sörgel, U., Hinz, S., 2020. Analysis of Airborne SAR and InSAR Data for Coastal Monitoring. 40. Wissenschaftlich-Technische Jahrestagung der DGPF in Stuttgart - Publikationen der DGPF, 29, 457-461.

Dorninger, P., 2011. Eine praktikable und genaue Methode zur Bestimmung von Wasser-Land-Grenzen aus LaserscannerDaten. Veranstaltungen 3/2011, Hrsg. Bundesanstalt für Gewässerkunde, Koblenz, Mai 2011, 93-100.

Horn, R., Jäger, M., Keller, M., Limbach, M., Nottensteiner, A., Pardini, M., Reigber, A., Scheiber, R., 2017. F-sar-recent upgrades and campaign activities. 2017 18th International Radar Symposium (IRS), IEEE, 1-10.

Pinheiro, M., Amao, J., Scheiber, R., Reigber, A., Schmitz, S., Thiele, A., Hinz, S., Bolz, T., Sörgel, U., Fritz, A., Müller, A., Havel, P., Brockmann, H., Wortmeyer, E., Dirks, H., Wurpts, A., 2020a. GeoWAM: Airborne SAR Interferometry for the Efficient Water Management in Coastal Areas affected by Tides.
40. Wissenschaftlich-Technische Jahrestagung der DGPF in Stuttgart - Publikationen der DGPF, 29, 457-461.

Pinheiro, M., Amao-Oliva, J., Scheiber, R., Jäeger, M., Horn, R., Keller, M., Fischer, J., Reigber, A., 2020b. Dual-Frequency Airborne SAR for Large Scale Mapping of Tidal Flats. Remote Sensing, 12(11), 1827.

Schmitz, S., Wortmeyer, E., Thiele, A., Dirks, H., Wurpts, A., 2021. Detection of mussel beds using Airborne polarimetric SAR data. Accepted for IGARSS 2021.

Sellerhoff, F., 2011. Aspekte der DGM-W-Modellierung in tidebeeinflussten Gebieten. Veranstaltungen 3/2011, Hrsg. Bundesanstalt für Gewässerkunde, Koblenz, Mai 2011, 70-81. 